\title{
Co-occurrence of multiple sclerosis and Thomsen's myotonia: a report of two cases
}

\author{
Fereshteh Ashtari, MDa \\ Seyed Amir Bahreini, PhD \\ Hamid Zahednasab, $\mathrm{PhD}^{\mathrm{c}}$
}

\begin{abstract}
a Department of Neurology, Isfahan Neurological Sciences Research Center, Isfahan Medical Education Research Center, Isfahan University of Medical Sciences, Isfahan, Iran

${ }^{b}$ Department of Human Genetics, Graduate School of Public Health, University of Pittsburgh, Pittsburgh, PA, USA

'Institute of Biochemistry and Biophysics, University of Tehran, Tehran, Iran
\end{abstract}

Correspondence to: Hamid Zahednasab E-mail: h.zahednasab@ut.ac.ir

\section{Summary}

Multiple sclerosis (MS) is a common demyelinating disease of the central nervous system in which an abnormal immune system response results in damage to the myelin sheaths surrounding nerves. The etiology of MS remains elusive, although it has been suggested to be influenced by both genetic and environmental factors.

Here, we report two exceptional cases of patients affected by both MS and Thomsen's myotonia. Electromyography and MRI scans confirmed the presence of both diseases in the referred cases. Although the mechanisms underlying the co-occurrence of MS and Thomsen's disease are unclear, we have endeavored to offer plausible explanations for it.

KEY WORDS: multiple sclerosis, Thomsen's myotonia, neuromuscular disease

\section{Introduction}

Multiple sclerosis (MS) is a chronic neuroinflammatory disease characterized by irreversible damage to the central nervous system. Although the underlying causes of MS are still unclear, an interaction of environmental and genetic factors is responsible for the appearance and development of the disease. Familial studies exploring the incidence of MS have suggested twin concordance rates of $3 \%$ and $26 \%$ among dizygotic and monozygotic twins, respectively (Ebers et al., 1986).

Thomsen's disease is the autosomal dominant form of myotonia congenita (MC); the disease has a childhood onset manifested by prominent myotonia and muscle cramps (Thomsen, 1876). Genetic studies have provided sufficient evidence for a role of muscle chloride channel gene (CLCN1) mutations in the development of MC (Parasivam et al., 2009). We here describe two patients affected by both MC and MS simultaneously.

\section{Case 1}

A 26-year-old man, who had developed MS some years previously, was referred to the Kashani Hospital MS Center. He had a relapsing-remitting disease course and recorded an Expanded Disability Status Scale (EDSS) score of 1.0 at the time of assessment. His first attack had been characterized by myelitis causing left leg paresis and urinary incontinence; he had experienced his second attack four years later, at the age of 15 years, presenting the symptoms of diplopia and left hemiparesis. However, exaggerated tendon reflexes and absence of abdominal reflexes, without weakness, were observed on the patient's neurological examination and his latest MRI showed inactive lesions in the brain (Fig. 1A).

Over time, the patient had been afflicted by MS relapses which had improved completely without any sequelae.

At the latest clinical examination, the patient showed muscle stiffness in the legs as well as an inability to open the eyelids after closing them tightly. These symptoms, characterized by the classic warm-up phenomenon, had appeared during his childhood. Examination showed myotonia after thenar muscle percussion. Electromyography (EMG) examination was performed and it exhibited the "dive bomber" sound indicating myotonia without myopathic pattern. The patient's conditions were clinically mild and did not induce disability.

Since Thomsen's disease is an inherited disorder, we analyzed this patient's family history (Fig. 2A). All blood parameters were normal or generally unremarkable. 


\section{Case 2}

The second case is a 34-year-old male affected by both relapsing-remitting MS and Thomsen's myotonia with an EDSS score of 1.5 at the time of evaluation. He was first seen by F.A. in 2006 with symptoms of paraparesis. MRI scans showed periventricular plaques in the brain (Fig 1B). Symptoms were relieved by high doses of corticosteroids. A few months later, the patient was again seen by F.A. for a tonic-clonic seizure and subsequently treated with carba-
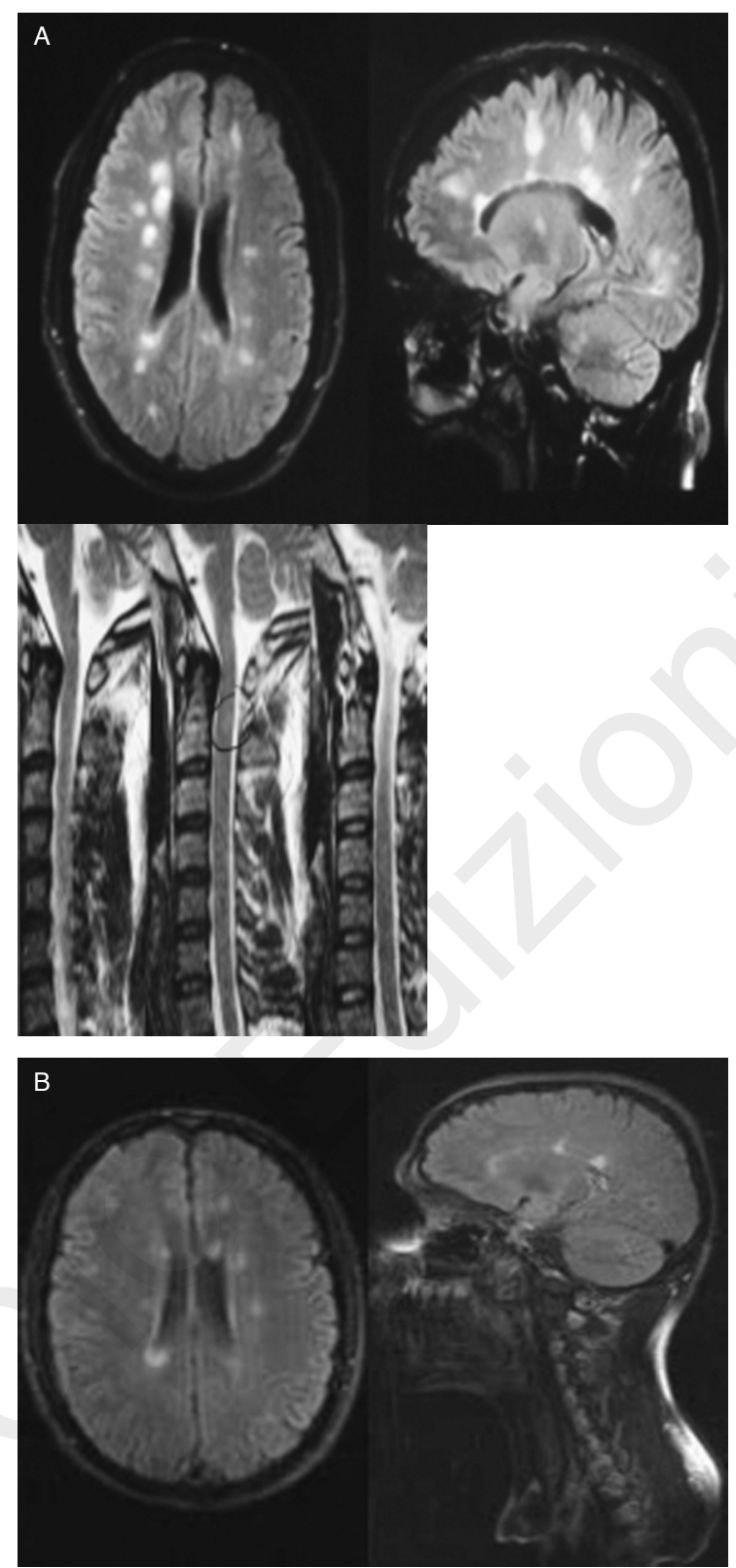

Figure 1 - The MRI scan of two MS patients suffering from Thomsen myotonia

(A) Cranial FLAIR MRI (left) and spine MRI (right) showing numerous demyelinating plaques in periventricular and centrum semiovale areas of the brain and white matter of the spinal cord. (B) Brain FLAIR MRI demonstrating numerous periventricular hypersignal lesions. mazepine. He has been treated with weekly intramuscular interferon injections since 2007. Over the past three years the patient has undergone a few sensory attacks and mild motor relapses. The patient's latest neurological examination showed bilateral Babinski's sign and absence of abdominal reflexes without weakness. The patient complained of muscle rigidity immediately after physical activity which was subsequently improved by exercise. In addition, the examination revealed percussion myotonia.

This patient was assessed for the presence of MC. EMG examination revealed a "dive bomber" sound indicating myotonia. It emerged that the patient has been afflicted with Thomsen's disease since childhood. The pedigree analysis demonstrated inheritance of $\mathrm{MC}$ in his family (Fig $2 \mathrm{~B}$ ). The results of the patient's blood tests showed no particular abnormality.

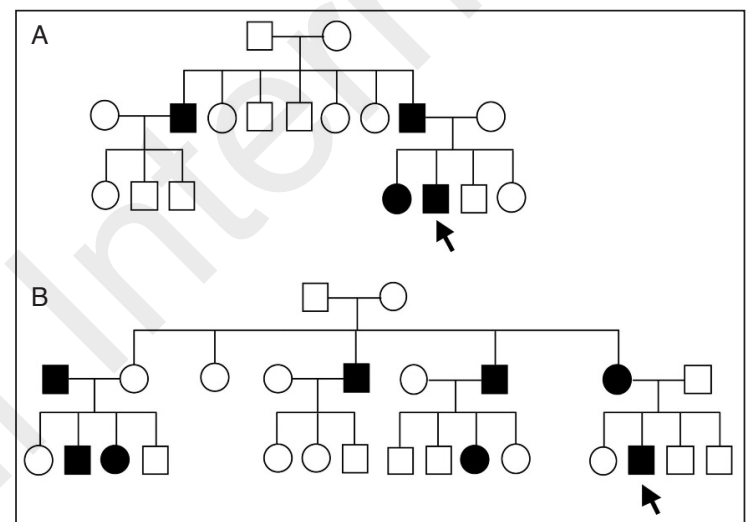

Figure 2 - The pedigrees of both patients show a dominant mode of inheritance indicating Thomsen form of myotonia congenita.

(A) Three patients with MC were detected in the first pedigree (case 1). No other MS patient was observed in this family. (B) Seven MC patients were diagnosed in the second pedigree (case 2). No members of this family were affected by MS.

\section{Discussion}

A myotonic-like syndrome in MS patients was described by Weintraub et al. (1970). Six years later Terrence (1976) reported the case of a 48-year-old man who was affected by both MS and myotonic dystrophy. More recently, other researchers (Parasivam et al., 2009; Portaro et al., 2013) have reported the cooccurrence of MS and Thomsen's disease. It would be important to understand whether the co-occurrence of MS and MC is coincidental or etiological. The present reports along with previous findings may undermine the assumption of a coincidental occurrence of MS and MC. However, large cohort studies are needed to test and validate these findings in terms of statistically significant associations.

$\mathrm{MC}$ is an inherited muscle disorder caused by mutations in the CLCN1 gene. MS is a complex disorder influenced by interactions between environmental factors and several genes, whose respective contributions remain to be clarified. The pedigrees of our patients 
show several other MC cases among their first- and second-degree relatives, even though there were no other reported cases of MS among their relatives.

Isfahan province in Iran is a high-risk region for the occurrence of MS, having a prevalence rate of $43.8 / 100,000$ (Saadatnia et al., 2007). Although the prevalence of $\mathrm{MC}$ has not yet been reported in Iran, an incidence rate of $1 / 100,000$ is estimated for this rare disorder, which is similar to its worldwide incidence (Farbu et al., 2003). If we consider the separate frequencies of these conditions, the incidence of co-occurrence of MS and MC would be $0.000438 / 100,000$. In other words, their casual co-occurrence is highly unlikely. This suggests that the presence of both conditions in our patients is probably not casual and may be linked to particular underlying mechanisms of pathogenesis.

The CLCN1 gene, consisting of 23 exons, lies on chromosome 7q35 (Rüdel and Lehmann-Horn, 1985). None of the linkage and genome-wide association studies conducted to date has detected a significant association between MS disease and the chr.7q35 region where the $C L C N 1$ gene is located. Given the genetic disparities between the two diseases it seems unlikely that MS and MC share similar genetic susceptibility. On the other hand, we should not overlook the "missing heritability" aspect of MS genetics; indeed, it should be pointed out that other susceptible loci are yet to be identified in MS. We believe that genetic and epigenetic factors play key roles in this scenario. There are several genetic polymorphisms within the CLCN1 gene not essentially leading to MC (Esteban et al., 1998; Sangiuolo et al., 1998) and could be of great interest for further analysis in MS patients.

\section{References}

Ebers GC, Bulman DE, Sadovnick AD, et al (1986). A population-based study of multiple sclerosis in twins. $N$ Engl $J$ Med 315: 1638-1642.

Esteban J, Neumeyer AM, McKenna-Yasek D, et al (1998). Identification of two mutations and a polymorphism in the chloride channel CLCN-1 in patients with Becker's generalized myotonia. Neurogenetics 1: 185-188.

Farbu E, Søfteland E, Bindoff LA (2003). Anaesthetic complications associated with myotonia congenita: case study and comparison with other myotonic disorders. Acta Anaesthesiol Scand 47: 630-634.

Parasivam S, Krupa M, Slee M, et al (2009). Clinical, electrophysiological and genetic features of a large Australian family with paramyotonia congenita. Med J Aust 190: 334-336.

Portaro S, Musumeci O, Rizzo V, et al (2013). Stiffness as a presenting symptom of an odd clinical condition caused by multiple sclerosis and myotonia congenita. Neuromuscul Disord 23:52-55.

Rüdel R, Lehmann-Horn F (1985). Membrane changes in cells from myotonia patients. Physiol Rev 65: 310-356.

Saadatnia M, Etemadifar M, Maghzi AH (2007). Multiple sclerosis in Isfahan, Iran. Int Rev Neurobiol 79: 357-375.

Sangiuolo F, Botta A, Mesoraca A, et al (1998). Identification of five new mutations and three novel polymorphisms in the muscle chloride channel gene (CLCN1) in 20 Italian patients with dominant and recessive myotonia congenita. Mutations in brief no. 118. Online. Hum Mutat 11: 331

Terrence CF (1976). Myotonic dystrophy and multiple sclerosis. J Neurol 213: 305-308.

Thomsen J (1876). Tonische Krämpfe in willkürlich beweglichen Muskeln in Folge von erebter psychischer Disposition (Ataxia muscularis?). Arch Psychiatr Nervenkr 6: 702-718.

Weintraub MI, Megahed MS, Smith BH (1970). Myotonic-like syndrome in multiple sclerosis. N Y State J Med 70: 677-679. 